\title{
Effect of Short Time Morphine Addiction on the Kidney Tissue in Rat
}

\author{
Reza Malekpourafshar and Hamid Zeinalinejad \\ Department of Pathology, Afzalipour Medical School, Kerman Medical Sciences University \\ Emam Khomeyni Highway, Kerman, Iran
}

\begin{abstract}
Opium is an important substance that abuses and has very pharmacological and pathological effects. Morphine is the main substance of opium. In the recent study, it has been tried to identify the short effects of morphine on the kidney pathology of Rats. The randomized double blind study was designed. Twenty rats were selected and divided in two groups and maintained under routine laboratory conditions and similar intervention. One group had been addicted orally by morphine powder and control were not addict. After three weeks, all rats were anesthetized and underwent autopsy. Their kidneys were removed en-block and sent to pathology laboratory and reviewed by one pathologist. The major considered variables were tubulo-interstitial and glomerular changes, glomerular amount and size on light microscope view. Glomerular, tubular and vascular changes, glomerular counts, glomerular size, and cortical thickness showed no significant differences between groups of study. Only Lymphocytic infiltration was higher frequency in addict group, significantly $p=0.007$. It seems short course of morphine consumption is very little effect on kidney, only Lymphocytic infiltration observe in addicted renal tissue which, may be precursor of other changes in kidney. It is better long term studies are implemented in this course.
\end{abstract}

Key words: Morphine, addict, kidney, rat

\section{INTRODUCTION}

In human and experimental animals, morphine is extensively metabolized via conjugative and oxidative pathways to metabolites that exhibit pharmacological activity $^{[1]}$. Although only $10 \%$ of a dose of morphine is excreted unchanged in urine. The kidneys play a major role in the excretion of its metabolites ${ }^{[2,3]}$. Drug metabolizing enzymes are known to exist in the mammalian kidney and morphine is one of many drugs that have been suggested to undergo renal metabolism. Clinical observations have shown higher level of renal disease in opium-addicted patients and there were many few studies about this subject. The effect of morphine on renal histopathology has been investigated rarely. The present study was designed to investigate the effect of morphine on microscopic renal tissue changes in the rats.

\section{MATERIALS AND METHODS}

A double blind randomized experimental trial was designed. Twenty male Spruge-Dawley rats with between 250-300 g were selected. They were divided to two groups randomly. One of them were addicted by morphine powder by rapid method of morphine dependency that examined by other researchers ${ }^{[4-8]}$. Morphine hydrochloride powder (Daru Pakhsh Ltd. Tehran-Iran) was used for addiction. After 3 weeks all cases and controls rats examined with Naloxane $2 \mathrm{mg} \mathrm{kg}^{-1}$ to check dependency. Signs of dependency were controlled by a check list ${ }^{[4-8]}$. Whereas rat exhibited dependency signs, were being taken for granted addict. Then they were anesthetized by Ketamine (Merk Co. Germany) and underwent autopsy. Their kidneys were removed en-block and sent to pathology laboratory in Formalin. After fixation process, pathologist provided paraffin based block of renal tissue and sliced them. Four kind of staining were performed for each specimens:

- Hematoxilin-Eosin for routine evaluation

- Periodic acid shift for evaluation of basement membrane changes

- Masson tri chromium staining for evaluation of tubulo-interstitial changes

- Jones staining for evaluation of glomerular and mesenchymal changes ${ }^{[9]}$.

All stained slices review from the viewpoint of tubulo-interstitial and glomerular changes, glomerular amount and size by light microscope with 400 in 5 fields.

Pathologist was blinded about groups and all blocks were review two times. The study process was approved by the committee of research at Kerman medical sciences university.

Corresponding Author: Reza Malekpourafshar, Department of Pathology, Afzalipour Medical School, Kerman Medical Sciences University, Emam Khomeyni Highway, Kerman, Iran Tel: 00989131405232 


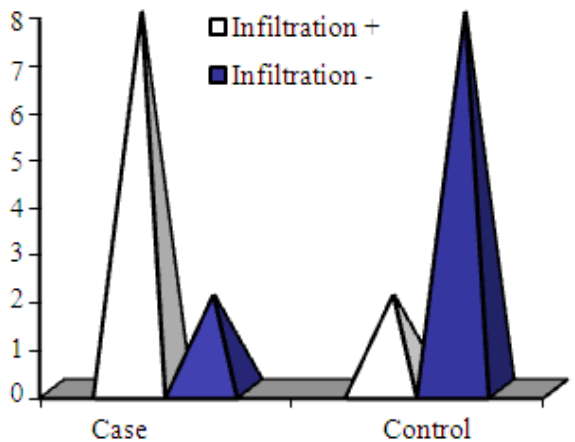

Fig 1: Frequency of lymphocyte infiltration in two groups, $\chi^{2}=7.2, \mathrm{DF}=1, \mathrm{p}=0.007$

For data analysis the SPSS 11.5 was used. Comparison of numeric variable like amount, size, and thickness of glomerous' was carried out by two sample t-test, and results were represented as mean+/-SD. Nominal data were compared between groups by Chi squared test. Difference was considered to be statistically significant at $\mathrm{p}<0.05$.

\section{RESULTS}

Among histopathological variables which were studied; glomerular, tubular and vascular changes showed no significant differences between groups of study.

In addict and control groups mean glomerular counts were $14.6+/-1.2$ and $15.5+/-0.7$ in order so the difference was not statistically significant $(\mathrm{p}=0.59)$.

Also mean cortical thickness was 208.8+/-27 micron in addict group and 178+/-15.5 micron in control group, Statistically no significant difference could be found between groups. The next variable which was analyzed was glomerular size in diameter. Mean glomerular diameter in addict and control groups were $25.4+/-1.8$ and $24.2+/-0.8$ which were not different statistically $(\mathrm{p}=0.85)$.

Lymphocytic infiltration in renal cortex was found in eight addict rats versus two rats in control group. The difference was statistically significant $(\mathrm{p}<0.007, \mathrm{DF}=1$, $\left.\chi^{2}=7.2\right)($ Fig. 1).

\section{DISCUSSION}

There are several substances, which are released from opium in the body. Morphine, which is the main alkaloid in this group, is thought to play the main role in what opium does in beings.

Morphine has several effects on physiology, histology and immune system of the body. Some of them, which are known, are listed below.

Weakening immune system, reduction of total body oxygen consumption and hypoxia, stimulating antidiuretic hormone release from kidneys, peripheral and visceral vasodilatation ${ }^{[10]}$.
Kidney is one of the main sites of morphine metabolism $^{[10]}$ so renal problems could be expected in morphine addiction. Johnson showed that application of morphine in rats could result in significant degenerative changes in kidneys. Increased pedocytic microinjections were one of the important changes in this study ${ }^{[11]}$.

Toupalik and coworkers showed that acute tubular nephritis is found in chronic heroin addicts and explained that this is the result of repeated hypoxia in kidneys $^{[12]}$.

Dettmeyer and coworkers found inflammatory reactions like lymphomonocytic glomerulonephritis. In a significant proportion of kidneys of addicts ${ }^{[13]}$.

Chronic tubulointrestitial disease with tubular atrophic changes and monocytic infiltration is another known problem, which is well described in chronic application of analgesics.

According to the high prevalence of opium addiction in our community and the clinical experiences that show a higher incidence of kidney problems in opium addicts comparing to non-addicts and also regarding the studies mentioned above we decided to design this study.

The differences of mean cortical thickness, glomerular count and glomerular diameter were not significant between two groups of study. But reversed variations like increased cortical thickness and glomerular diameter con-committed with decreased glomerular count in addicts can show primary stages of a renal pathology.

Kidneys respond to stress by hypertrophy but not hyperplasia. According to these reversed variations and just slightly high p-values 0.06 , and 0.059 . One may conclude that sample size or addiction duration could be the reason. As mentioned above duration of addiction could be important. In this study, we found primary stages of renal pathology .Maybe continuing the study could reveal and show the differences between two groups.

Something important found in this study is significantly higher proportion of rats with lymphocytic infiltration in kidneys among addict rats comparing to the non-addict ones. This could show presentation of kidney problems in addict rats. The most important factor responsible for this is hypoxia in kidneys. As mentioned before, morphine could decrease systemic and therefore renal blood flow by peripheral and visceral vasodilatation ${ }^{[10]}$. Decreased total oxygen consumption is another factor, which could increase hypoxia. Kidney is sensitive to hypoxia and responds it by infiltration of inflammatory cells.

\section{CONCLUSION}

Anyhow, we recommend more studies in cellular and molecular levels and different addiction durations 
to be done in order to show what are the changes caused by morphine.

\section{REFERENCES}

1. Milne, R.W., R.L. Nation and A.A. Somogyi, 1996. The disposition of morphine and 3-and 6glucoronide metabolites in humans and animals and the importance of the metabolites to pharmacological effects of morphine. Drug Metab. Rev., 28: 345-372.

2. Milne, R.W., R.L. Nation, A.A. Somogyi, F. Bochner and W.M Griggs, 1992. The influence of renal function on the renal clearance of morphine and its metabilites in intensive care patients. $\mathrm{Br}$. J. Pharmacol., 34: 53-59.

3. Somogyi, A.A., R.L Nation, C. Olweny and et al., 1993. Plasma concentration and renal clearance of morphine, morphine 3-glucoronide and morphine 6-glucoronide in cancer patients receiving morphine. Clin. Pharmacokinet., 24: 413-420.

4. Badway, A.A., C.M. Evans and M. Evans, 1982. Production of tolerance and physical dependency in rats by simple administration of morphine in drinking water. Br. J. Pharmacol., 75: 485-491.

5. Leung, C.M. and C.W. Diasandogle, 1986. Rapid induction to dependence to morphine in rats. Neuropharmacology, 25: 305-307.

6. Rezaee-Gharaee, L. and M. Sabetkasaee, 1990. Comparison of postoperative pain in morphine addicted rats. Iran. Physiol. Pharmacol., 5: 153-160.

7. Karimi-Mobarake, M., R. Malekpourafshar and M. Shamsimeymandi, 2003. Effect of morphine dependency to bone healing in rats. J. Kerman Med. Sci. Univ., 11: 1-7.
8. Gullert, V.F. and S.G. Holtzman, 1978. Development and maintenance of morphine tolerance and dependence in the rat by scheduled access to morphine drinking solutions. J. Pharmacol. Exp. Ther., 205: 539-546.

9. Ordonez, N.G., 2004. Urinary Tract. In: Rosai and Akerzman's Surgical Pathology, Rosai J. (Ed.), 9th Edn., Mosby Inc., St. Louis, USA., pp: 163-182.

10. Way, W.L., H.L. Field and M.A. Schumacher, 2001. Opioid Analgesics and Antagonists. In: Basic and Clinical pharmacology, Katzung, B.G. (Ed.), 8th Edn., McGraw Hill Co., New York, USA., pp: 512-531.

11. Johnson, J.E., J.J. White and R.C. Walovitch, 1987. Effects of morphine on rat kidney glomerular podocyte. Drug. Alcoh. Depend., 19: 249-53.

12. Toupalik, P., H. Vanerkova, P. Klir and I. Bouska, 2002. Morphologic findings in chronic abuse of heroin and pervitine. Soud. Lek., 47: 5-11.

13. Dettmeyer, R., G. Stojanovski and B. Madea, 2000. Pathogenesis of heroin associated glomerulonephritis. Correlation between the inflammatory activity and renal deposits of immunoglobulin and complement? Forensic. Sci. Int., 113: 227-231. 